\title{
Clinical features and treatment outcomes of blastic plasmacytoid dendritic cell neoplasm: a single- center experience in Korea
}

\author{
Hae Su Kim ${ }^{1,2}$, Hee-Jin Kim³ ${ }^{3}$ Sun-Hee Kim³ ${ }^{3}$ Joon Young Choi ${ }^{4}$, Young Hyeh $\mathrm{Ko}^{5}$, Won Seog Kim ${ }^{1}$, \\ Chul Won Jung ${ }^{1}$, and Seok Jin Kim ${ }^{1,6}$
}

\begin{abstract}
${ }^{1}$ Division of Hematology-Oncology, Department of Medicine, Samsung Medical Center, Sungkyunkwan University School of Medicine, Seoul; ${ }^{2}$ Division of HematologyOncology, Department of Medicine, Veterans Health Service Medical Center, Seoul; Departments of ${ }^{3}$ Laboratory Medicine \& Genetics, ${ }^{4}$ Nuclear Medicine, and ${ }^{5}$ Pathology, Samsung Medical Center, Sungkyunkwan University School of Medicine, Seoul; ${ }^{6}$ Department of Health Sciences and Technology, Samsung Advanced Institute for Health Sciences \& Technology, Sungkyunkwan University, Seoul, Korea
\end{abstract}

Received: December 20, 2015 Revised : March 11, 2016 Accepted: March 17, 2016

\section{Correspondence to}

Seok Jin Kim, M.D.

Division of Hematology-

Oncology, Department of

Medicine, Samsung Medical

Center, Sungkyunkwan University School of Medicine, 81 Irwon-ro, Gangnam-gu, Seoul 06351, Korea

Tel: $+82-2-3410-1766$

Fax: +82-2-3410-1754

E-mail:kstwoh@skku.edu
Background/Aims: Blastic plasmacytoid dendritic cell neoplasm (BPDCN) is a rare hematologic malignancy that typically presents in the form of skin manifestations with or without lymph node and bone marrow involvement. Given its rarity and recent recognition as a distinct pathological entity, no standard of treatment exists for this aggressive disease and its prognosis is particularly dismal.

Methods: We retrospectively analyzed clinical features and treatment outcomes of patients who were diagnosed with BPDCN between 2000 and 2014.

Results: Ten patients had a median age at diagnosis of 41 years (range, 18 to 79), and seven patients were male. Sites of disease involvement were the skin $(n=7)$, lymph node $(\mathrm{n}=5)$, bone marrow $(\mathrm{n}=2)$, liver $(\mathrm{n}=2)$, spleen $(\mathrm{n}=2)$, and soft tissue ( $\mathrm{n}$ $=1$ ). Intensified chemotherapy regimens such as hyperCVAD regimen (cyclophosphamide, vincristine, doxorubicin, dexamethasone, methotrexate, cytarabine), and VPDL (vincristine, methylprednisolone, daunorubicin, L-asparaginase) were used as a first-line treatment. Although all patients treated with intensified chemotherapy showed an objective response (five patients with complete response) with median progression-free survival of 11.2 months (range 6.2 to 19.4), complete remission was not sustained for more than 2 years in any case. The response was relatively long-lived compared with previously reported CHOP (doxorubicin, cyclophosphamide, vincristine, prednisone)-like regimens, but the above regimens do not result in long-term remission.

Conclusions: All patients treated with hyperCVAD or VPDL showed an objective response, but the duration of response was relatively short. Thus, the development of more effective induction as well as consolidation treatment strategy should be warranted to improve this rare disease entity.

Keywords: Blastic plasmacytoid dendritic cell neoplasm; Therapeutics; Survival outcome

\section{INTRODUCTION}

Blastic plasmacytoid dendritic cell neoplasm (BPDCN), previously referred to as blastic natural killer cell lym- phoma or $\mathrm{CD}_{4+} / \mathrm{CD}_{5} 6+$ hematodermic neoplasm, is a rare hematologic malignancy that is derived from plasmacytoid dendritic cells (PDCs) [1-4]. BPDCN, which was categorized as an "acute myeloid leukemia and related 
precursor neoplasm" by the World Health Organization (WHO) in 2008, is typically characterized by $\mathrm{CD}_{4}+$ and $\mathrm{CD} 56+$ co-expression without common lymphoid or myeloid lineage markers, suggesting derivation from PDC precursor $[1,3,5]$. Since the first case was described, approximately 200 cases have been reported due to its extremely low incidence [6-8]. This disease has a tendency to involve the skin, lymph nodes, peripheral blood, and bone marrow [7,9], and the cutaneous involvement is a predominant clinical feature of BPDCN ranging in appearance from small bruise-like areas to patches, nodules, and ulcerated masses [3,9,10]. In spite of its indolent nature confined to the skin without any constitutional symptoms, radiotherapy was found to be ineffective because most patients eventually relapse [11]. Although a substantial number of patients respond to initial chemotherapy like lymphoma patients, the outcome of systemic chemotherapy also has been disappointing because of its relatively short duration of response $[9,10,12]$. As a result, the optimal treatment strategy for BPDCN has not been established because the outcome of various chemotherapy regimens is not satisfactory. As a current clinical practice, intensified chemotherapy regimens have been used as a primary treatment for BPDCN in conjunction with the consolidation treatment such as autologous or allogeneic stem cell transplantation (SCT). However, there are limited data on the outcomes of these treatment approaches, especially Asian patients with BPDCN [13-15]. Herein, we reported our single-center experience with BPDCN patients receiving various chemotherapy regimens.

\section{METHODS}

\section{Patients}

We queried the terms 'blastic plasmacytoid dendritic cell neoplasm' and 'blastic natural killer cell lymphoma' in biopsy reports in the Samsung Medical Center electronic medical records database from January 1, 2000 to June 30, 2014. Through this process, adult patients (age $\geq 18$ years) whose medical records contained these terms were selected. After we manually reviewed the medical records of these patients, we selected 10 patients who were consecutively diagnosed with BPDCN. Those cases were reviewed by an expert pathologist for lymphoma according to the diagnostic criteria of the WHO 2008 classification [5]. Morphological analysis of skin and breast biopsies was conducted. All immunostains were performed on formalin-fixed, paraffin-embedded specimens. Immunohistochemical (IHC) criteria for diagnosis of BPDCN were expression of $\mathrm{CD}_{4}$ and $\mathrm{CD}_{5} 6$ by blastic tumor cells, coupled with at least on plamacytoid dendritic cell-specific antigen among CD123 or terminal deoxynucleotidyl transferase (TdT), in the absence of lineage-specific markers for B cells (CD2O), T cells (CD3), monocytes (lysozyme), and myeloid cells (myeloperoxidase [MPO]). Clinical data obtained included sex, age, initial presentation, biopsy site, initial manifestation, laboratory findings at diagnosis, treatment, and outcomes. This retrospective study was approved by the Institutional Review Board of Samsung Medical Center (IRB No: 2015-12-021) for the release of case information that was rendered anonymous. Thus, obtaining an informed consent was waivered.

\section{Treatment review and survival analysis}

Since 2000, various chemotherapy regimens have been used for BPDCN at our institution according to physician's preference. The most commonly used regimen was the hyperCVAD regimen (course A: cyclophosphamide, vincristine, doxorubicin, dexamethasone; course B: methotrexate, cytarabine) chemotherapy, and L-asparaginase-containing chemotherapy regimens including VPDL (vincristine, methylprednisolone, daunorubicin, L-asparaginase), and VIDL (etoposide, ifosfamide, dexamethasone, L-asparaginase). Thus, we compared patients according to the use of anthracycline-based intensified regimen and L-asparaginase-containing intensified regimen. Because all patients showed relapse or progression after their initial treatments, salvage chemotherapy regimens were also used including ESHAOX (etoposide, methylprednisolone, oxaliplatin, cytarabine), GDP (gemcitabine, dexamethasone, cisplatin), and SMILE (methotrexate, etoposide, ifosfamide, dexamethasone, L-asparaginase). We also analyzed the response to these salvage treatments based on the relevant response criteria. Thus, for patients diagnosed with BPDCN after 2007, ${ }^{18} \mathrm{~F}$-fluorodeoxyglucose (FDG) positron emission tomography/computed tomography (PET/CT) was used according to the revised response criteria for malignant lymphoma whereas the response of patients was 
evaluated by the International Working Group criteria before 2007. Autologous or allogeneic SCT were also performed as a consolidation treatment for responders to chemotherapy after complete response (CR) to initial therapy or salvage treatment if patients were eligible for SCT. The last survival status update was done at the time of analysis in September 2015. Survival curves were generated by the Kaplan-Meier method and compared using the log-rank test. Overall survival(OS) was calculated from the date of diagnosis to the date of death resulting from any cause. The time to progression was the date of diagnosis to the date of documented disease progression or relapse.

\section{RESULTS}

\section{Patient characteristics}

The study included seven male and three female patients with a median age at diagnosis of 41 years (range, 18 to 79 ). All patients had single or multiple skin lesions at presentation, and half of patients had multiple lymph node enlargements (Table 1). Cutaneous lesions were morphologically variable and involved the face, trunk, and extremities (Fig. 1). Six patients had a solitary cutaneous lesion, while the other patients had multiple skin lesions (Table 1). The median time between the onset of lesions and pathologic diagnosis was 2.6 months (range, 0.7 to 6.6). Bone marrow involvement was found in three patients at diagnosis, and they all showed abnormal findings in peripheral blood (Table 1). Two patients had hepatosplenomegaly, and both presented with thrombocytopenia. Only one patient (\#4) experienced B symptoms including weight loss and chills at diagnosis. Half of patients showed elevated levels of serum LDH; however, other laboratory values including creatinine and albumin were within normal range. All but one patient were diagnosed with BPDCN without any relevant disease history. One patient (\#9) previously had myelodysplastic syndrome (refractory anemia with excess blasts-2), and received decitabine treatment followed by allogenic hematopoietic SCT with fludarabine and busulfan conditioning. Twenty-two months later, he presented with cheek swelling and was diagnosed with BPDCN.

\section{Pathology}

For pathologic diagnosis, seven patients underwent skin biopsy, one patient underwent breast mass and axillary lymph node biopsies, one lymph node biopsy, and one periorbital and maxillary mass biopsies. On immunohistochemistry, patients showed $\mathrm{CD}_{4}, \mathrm{CD}_{5} 6$, and $\mathrm{CD}_{12} 3$ positivity, and negativity of $\mathrm{CD}_{3}, \mathrm{CD}_{2} \mathrm{O}$, and MPO in tumor cells (Table 2). Conventional cytogenetic studies with bone marrow aspirates at diagnosis indicated that three patients had abnormal cytogenetics (Table 2). The findings of ${ }^{18} \mathrm{~F}$-FDG PET/CT were available in seven patients. Of these patients, the FDG uptake of lesions were variable, and this uptake was minimal in patients with only cutaneous manifestations. The percentage of Ki67 was relatively low in most cases, similar to indolent lymphomas.

\section{First-line treatment and outcome}

Four patients received hyperCVAD chemotherapy as a first-line treatment, and three patients showed a CR, whereas one patient showed a partial response (PR). Among them, the patient with a PR (\#2) received adjuvant radiotherapy for breast mass and axillary lymph nodes. However, she developed local relapse 6 months later. Another patient (\#7) with CR underwent upfront allogenic SCT from a fully matched sibling donor after treatment with 10 Gy total body irradiation conditioning and cyclophosphamide $60 \mathrm{mg} / \mathrm{kg}$ body weight. He maintained a CR for 14 months, but ultimately relapsed with multiple skin lesions. In particular, leukemic transformation was found in one patient (\#1) at the time of relapse 19 months after first-line treatment (Table 3). One patient initially treated with CHOP (doxorubicin, cyclophosphamide, vincristine, prednisone) also showed disease progression after she achieved PR (\#10). Three patients received L-asparaginase-containing chemotherapy regimens including VPDL and VIDL. Although they all responded to the treatment, they all relapsed (\#3, \#5, and \#6) (Table 3). One patient (\#9) received palliative radiation therapy to the maxilla, but his periorbital mass progressed. The other patient (\#4) did not receive any treatment with curative intent because of advanced age and comorbidity.

\section{Salvage treatment and outcome}

After relapse or disease progression occurred, second-line 


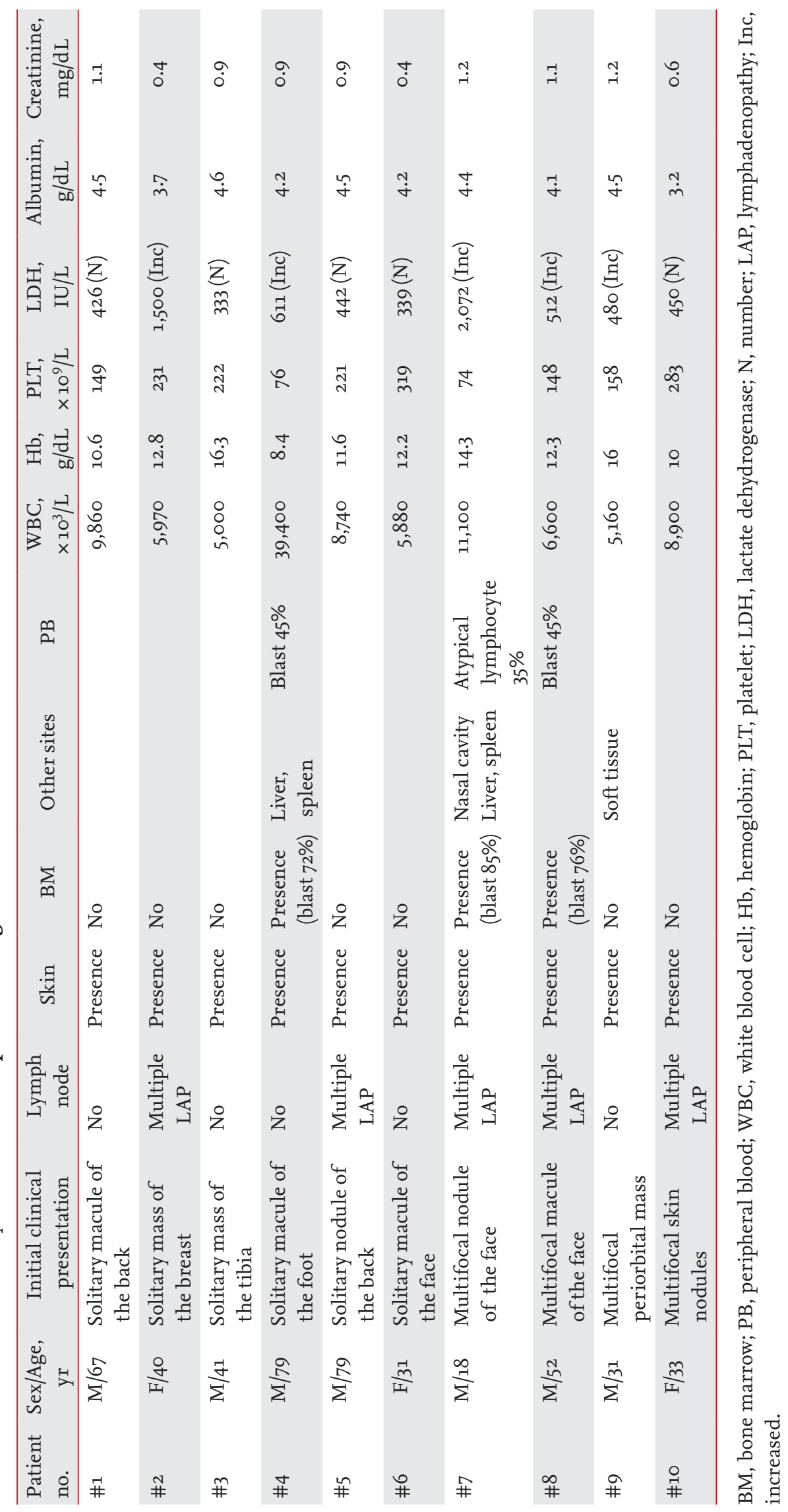




\section{KJIM ${ }^{-}$}
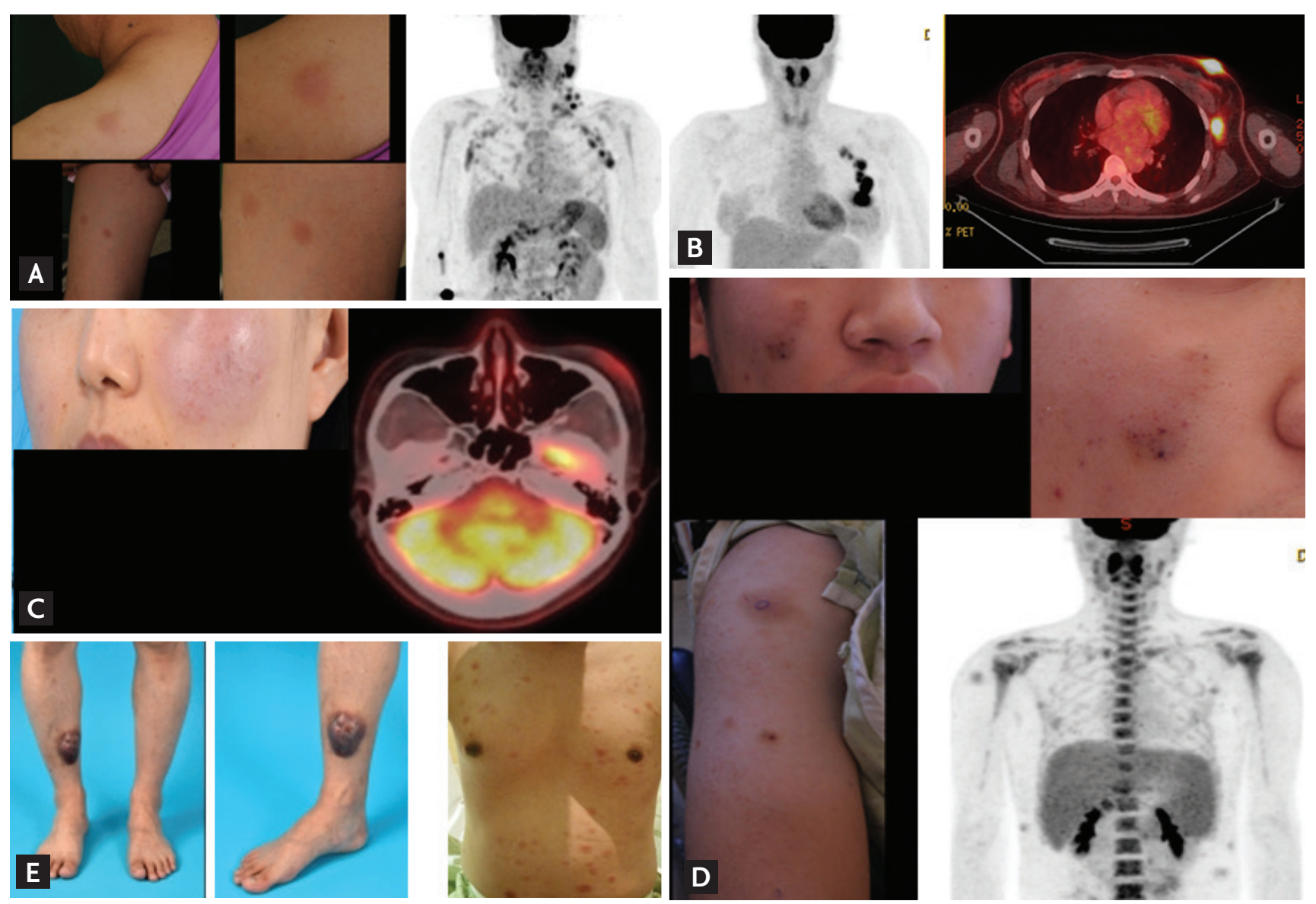

D

Figure 1. Initial presentation of patients. (A) Patient (\#8) presented with multiple skin lesions and lymph node enlargement on positron emission tomography/computed tomography. (B) Patient (\#2) displayed breast skin lesions with increased fluorodeoxyglucose (FDG) uptake and axillary lymph node enlargement. (C) Patient (\#6) showed a localized skin lesion of the cheek with moderate FDG uptake. (D) Patient (\#7) presented with multiple skin lesions and hepatosplenomegaly. (E) Patient (\#9) initially showed a solitary skin lesion of the lower leg (left); however, multiple lesions developed on the trunk, indicating relapse (right).

treatment with combination chemotherapy, local radiotherapy, or autologous SCT were applied. The regimens used included ESHAOX, GDP, VPDL, and SMILE. Most patients responded poorly to second-line therapies; however, one patient (\#8) was salvaged with four cycles of ESHAOX, and underwent autologous SCT. Although he maintained the 2nd CR for 7 months, he relapsed and died due to disease progression (Table 3). Another patient (\#1) achieved a PR after completion of the ist cycle of VPDL; however, he immediately progressed to leptomeningeal involvement and subsequently died. Interestingly, patient \#2 who presented with a breast mass and multiple involved lymph nodes received SMILE, DHAP (cisplatin, cytarabine, dexamethasone), and GDP as salvage chemotherapy, but she did not respond to treatment. Finally she underwent intensified chemotherapy with MEC (mitoxantrone, etoposide, cytara- bine), and remains in CR without any signs of relapse.

\section{Survival outcome}

The median OS of patients was 34 months, and five patients were alive at the time of analysis (Fig. 2A). All patients experienced at least one relapse event or progression regardless of whether they had a single or multiple skin lesions at diagnosis, with a median progression-free survival of 11.2 months (range, 6.2 to 19.4). The pattern of relapse was also variable; thus, some patients showed repeated local relapse (e.g., patient \#2), while other patients showed distant relapse (e.g., patient \#1 and \#5). Thus, the initial presentation of skin lesions was not significantly associated with OS (Fig. 2B). Although one patient who underwent SCT did not show evidence of relapse, she died due to transplantation-re- 


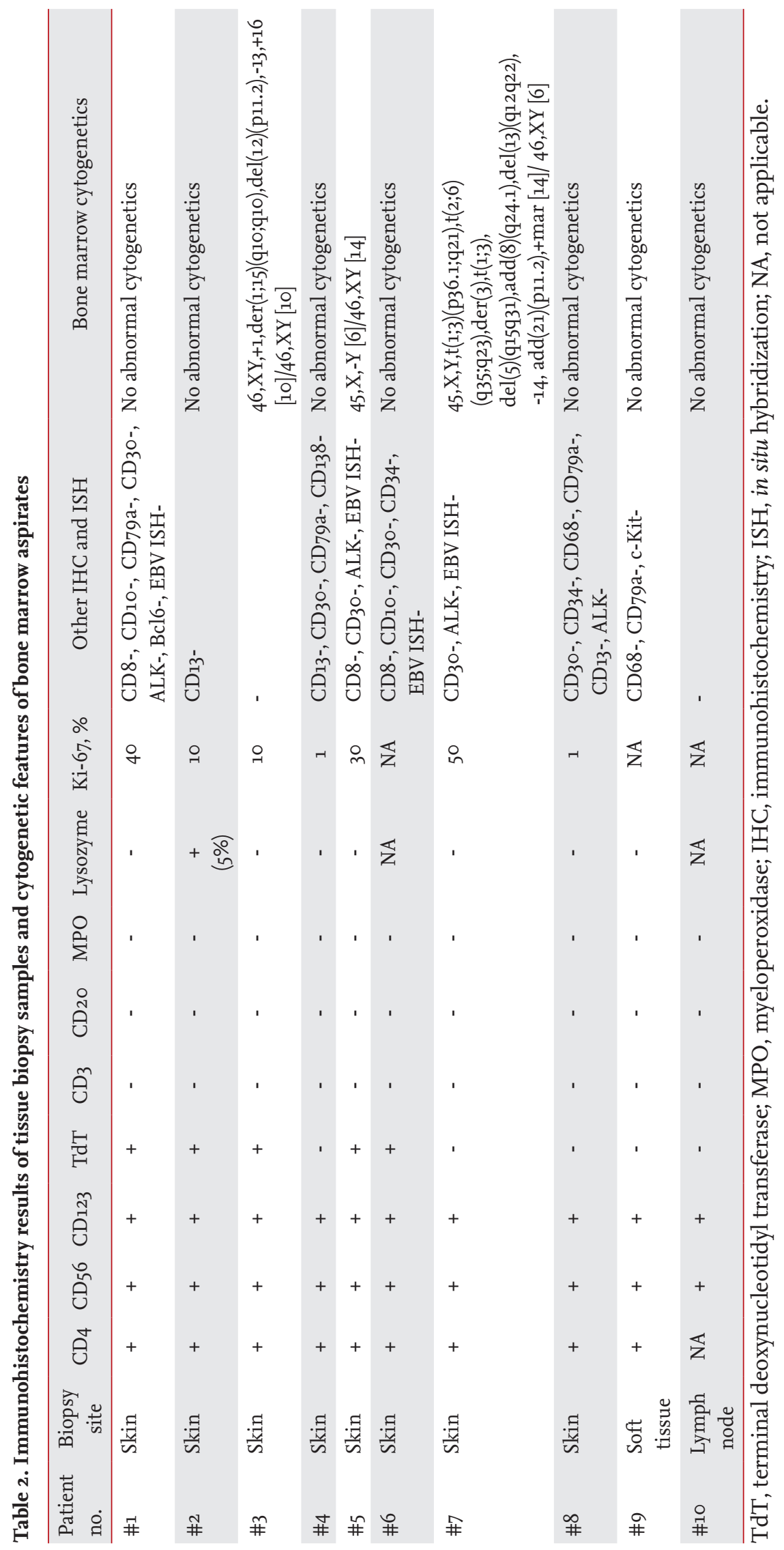


Table 3. Treatment and clinical course of patients

\begin{tabular}{|c|c|c|c|c|c|c|}
\hline $\begin{array}{l}\text { Patient } \\
\text { no. }\end{array}$ & $\begin{array}{l}\text { First treatment } \\
\text { (cycle or Gy) }\end{array}$ & $\begin{array}{l}\text { Time to } \\
\text { progression, } \\
\text { mon }\end{array}$ & $\begin{array}{l}\text { Salvage treatment } \\
\text { (cycle or Gy) }\end{array}$ & $\begin{array}{l}\text { Clinical course after } \\
\text { salvage treatment }\end{array}$ & $\begin{array}{l}\text { Overall } \\
\text { survival, } \\
\text { mon }\end{array}$ & $\begin{array}{c}\text { Survival } \\
\text { status }\end{array}$ \\
\hline$\# 1$ & $\begin{array}{l}\text { HyperCVAD (\#6) } \\
\rightarrow \mathrm{CR} \rightarrow \mathrm{PD} \\
\text { (leukemic } \\
\text { transformation) }\end{array}$ & 19 & $\begin{array}{l}\mathrm{VPDL}(\# 1) \\
\rightarrow \mathrm{PR} \rightarrow \mathrm{PD}\end{array}$ & $\begin{array}{l}\text { Disease progression } \\
\text { in CNS }\end{array}$ & 23 & Dead \\
\hline$\# 2$ & $\begin{array}{l}\text { HyperCVAD (\#6) + } \\
\mathrm{RT}(40 \mathrm{~Gy}) \\
\rightarrow \mathrm{PR} \rightarrow \mathrm{PD}\end{array}$ & 6 & $\begin{array}{l}\operatorname{SMILE}(\# 1) \\
\rightarrow \mathrm{PD} \rightarrow \mathrm{DHAP}(\# 2) \rightarrow \mathrm{PD} \\
\rightarrow \mathrm{GDP}(\# 2) \rightarrow \mathrm{PD}\end{array}$ & $\begin{array}{l}\text { MEC chemotherapy } \\
\rightarrow \text { CR }\end{array}$ & 25 & Alive \\
\hline \#3 & $\begin{array}{l}\mathrm{VPDL}(\# 3) \\
\rightarrow \mathrm{CR} \rightarrow \mathrm{PD}\end{array}$ & 11 & $\begin{array}{l}\mathrm{RT}(40 \mathrm{~Gy}) \\
\rightarrow \mathrm{PR} \rightarrow \mathrm{GDP}(\# 3) \rightarrow \mathrm{CR}\end{array}$ & $\begin{array}{l}\text { Ongoing } \\
\text { chemotherapy }\end{array}$ & 20 & Alive \\
\hline \#4 & Not done & NA & NA & NA & 6 & Dead \\
\hline \#5 & $\begin{array}{l}\mathrm{VIDL}(\# 2) \\
\rightarrow \mathrm{CR} \rightarrow \mathrm{PD}\end{array}$ & 3 & $\begin{array}{l}\mathrm{RT}(30 \mathrm{~Gy}) \\
\rightarrow \mathrm{PD}\end{array}$ & $\mathrm{GDP}(\# 2) \rightarrow \mathrm{PD}$ & 6 & Dead \\
\hline \#6 & $\begin{array}{l}\mathrm{VPDL}(\# 2) \\
\rightarrow \mathrm{PR} \rightarrow \mathrm{PD}\end{array}$ & 4 & SMILE $(\# 2) \rightarrow$ PR & $\begin{array}{l}\text { Ongoing } \\
\text { chemotherapy }\end{array}$ & 9 & Alive \\
\hline \#7 & $\begin{array}{l}\text { HyperCVAD }(\# 4)+ \\
\text { Allo-SCT } \\
\rightarrow \mathrm{CR} \rightarrow \mathrm{PD}\end{array}$ & 14 & $\begin{array}{l}\mathrm{GDP}(\# 4) \\
\rightarrow \mathrm{PR} \rightarrow \mathrm{PD}\end{array}$ & $\mathrm{VIDL} \rightarrow \mathrm{PR}$ & 24 & Alive \\
\hline \#8 & $\begin{array}{l}\text { HyperCVAD }(\# 2) \\
\rightarrow \mathrm{CR} \rightarrow \mathrm{PD}\end{array}$ & 7 & $\begin{array}{l}\text { ESHAOX }(\# 4)+\text { Auto-SCT } \\
\rightarrow \mathrm{CR} \rightarrow \mathrm{PD}\end{array}$ & $\begin{array}{l}\text { MEC chemotherapy } \\
\rightarrow \text { PD }\end{array}$ & 18 & Dead \\
\hline \#9 & $\begin{array}{l}\mathrm{RT}(21 \mathrm{~Gy}) \\
\rightarrow \mathrm{PR} \rightarrow \mathrm{PD}\end{array}$ & 2 & $\begin{array}{l}\mathrm{RT}(36 \mathrm{~Gy}) \\
\rightarrow \mathrm{SD} \rightarrow \mathrm{PD}\end{array}$ & IA chemotherapy & 19 & Alive \\
\hline$\# 10$ & $\begin{array}{l}\mathrm{CHOP}(\# 6) \\
\rightarrow \mathrm{PR} \rightarrow \mathrm{PD}\end{array}$ & 12 & $\begin{array}{l}\mathrm{DHAP}(\# 2) \rightarrow \mathrm{PD} \\
\rightarrow \text { CALGB }+ \\
\text { Allo-SCT } \rightarrow \text { CR }\end{array}$ & $\begin{array}{l}\text { Chronic GVHD with } \\
\text { pneumonia during } \\
\text { follow-up } \rightarrow \text { Death }\end{array}$ & 34 & Dead \\
\hline
\end{tabular}

Gy, gray; HyperCVAD, alternate cycles of hyperfractionated cyclophosphamide, vincristine, doxorubicin, dexamethasone, and methotrexate, cytarabine; CR, complete response; PD, progression disease; VPDL, vincristine, methylprednisolone, daunorubicin, L-asparaginase; PR, partial response; CNS, central nervous system; RT, radiation therapy; SMILE, methotrexate, etoposide, ifospamide, dexamethasone, L-asparaginase; DHAP, cisplatin, cytarabine, dexamethasone; GDP, gemcitabine, dexamethasone, cisplatin; MEC, mitoxantrone, etoposide, cytarabine; NA, not applicable; VIDL, vincristine, etoposide, ifosfamide, dexamethasone; Allo-SCT, allogenic stem cell transplantation; ESHAOX, etoposide, methylprednisolone, oxaliplatin, cytarabine; Auto-SCT, autologous stem cell transplantation; SD, stable disease; IA, idarubicin, cytarabine; CHOP, doxorubicin, cyclophosphamide, vincristine, prednisone; CALGB, cyclophosphamide, daunorubicin, vincristine, prednisone; GVHD, graftversus-host disease.

lated complications (pneumonia and chronic graft-versus-host disease).

\section{DISCUSSION}

This rare but fatal disease entity was first found to originate from the precursor of PDCs in 1999, and the final diagnosis criteria for BPDCN were determined in the 2008 version of the WHO classification of tumors of hematopoietic and lymphoid tissue $[16,17]$. A previ- ous study analyzing the cutaneous presentation of 90 patients with BPDCN described 66 patients (73\%) who presented with nodular lesions, 11 patients (12\%) with 'bruise-like' patches, and 13 (14\%) with disseminated lesions (patches and nodules) [10]. Consistent with these findings, all patients in our study had cutaneous lesions including two patients with nodules, four with macules, and two with masses. Pathologically, BPDCN shows abundant cytoplasm with a low or medium nucleus to cytoplasmic ratio and displays basophilia without granulation; diagnosis is based on the immunophenotypic 

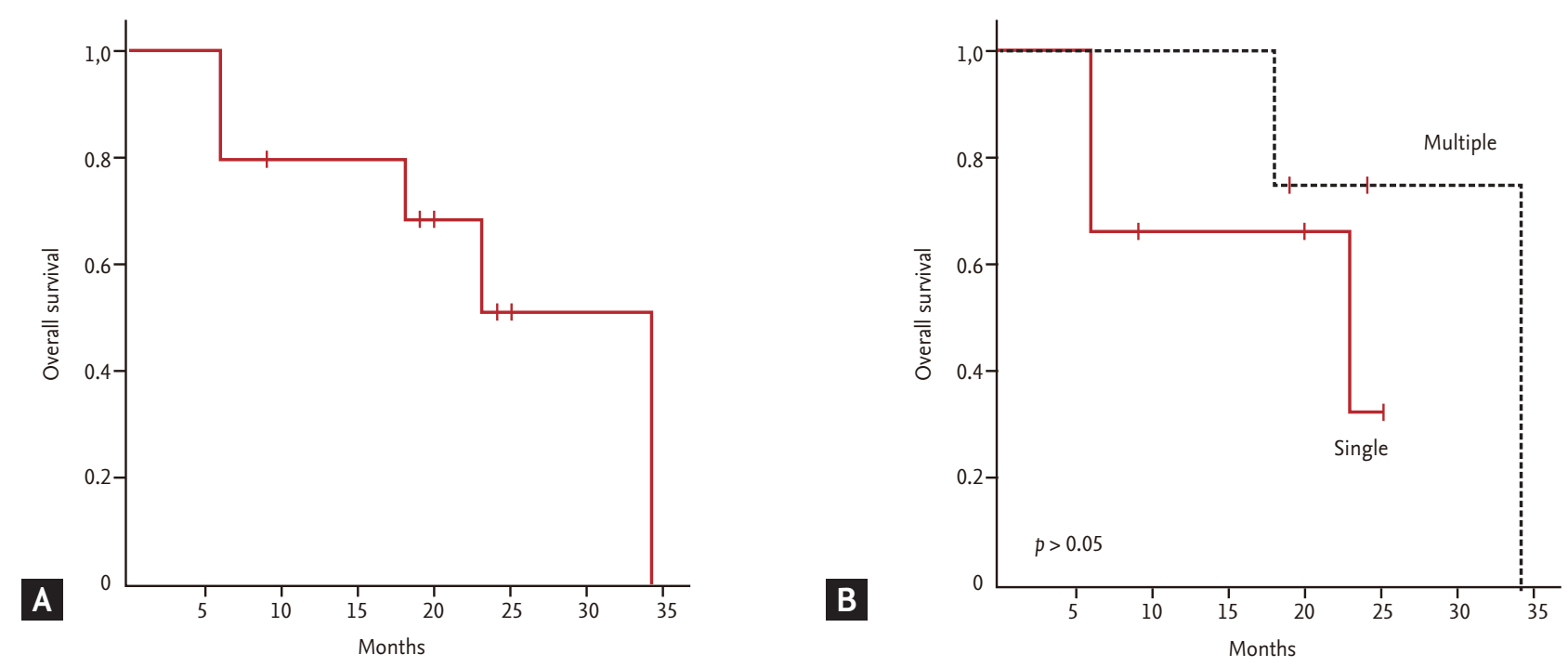

Figure 2. (A) Overall survival. (B) Comparison of overall survival according to the number of skin lesions at diagnosis.

profile [9]. Given its rarity and only recent recognition as a distinct clinicopathologic entity, there is no consensus on the immunohistochemistry markers for diagnosis of BPDCN $[9,10]$. However, a previous study of 91 patients using a panel of 12 IHC markers identified five markers (CD4, CD56, CD123, CD103, and TCL1) that could be important for diagnosis [10]. Thus, a typical case of BPDCN could co-express $\mathrm{CD}_{4}, \mathrm{CD}_{5} 6$, and $\mathrm{CD}_{123}$ without expressing common lymphoid or myeloid lineage markers $[8,18]$. A karyotyping study demonstrated that cytogenetic changes were complex, mainly involving chromosomes 5, 9, 12, 13, and 15 [19]. In our study, all patients expressed $\mathrm{CD}_{4}, \mathrm{CD}_{5} 6$, and $\mathrm{CD}_{123}$. Among these, eight patients did not express common lymphoid or myeloid lineage markers such as $\mathrm{CD}_{3}$, MPO, and lysozyme. Only lysozyme stained positively in $5 \%$ of tumor cells in one patient. Cytogenetic abnormalities were observed in three patients, while seven patients displayed a normal karyotype.

Because there is no consensus on the optimal therapy for BPDCN, therapeutic approaches vary widely from radiation therapy in localized stages to myeloablative therapy. Reimer and colleagues [20] reported that a CHOPlike regimen was used most frequently, and the overall response rate in 38 patients was about $71 \%$ (CR, 55\%; PR, $16 \%)$. However, accumulated data showed that radiation therapy or CHOP-like regimens failed to sustain CR status [7,11]. Dalle et al. [11] reported that CHOP-like regimens frequently induce disease remission; however, the duration of response was only around 5 months. In general, ALL/lymphoma-type regimens including hyperCVAD and VPDL were reported to show better survival outcomes than AML-type regimens [12,21]. Thus, in a previous study of 41 patients, the CR rates of ALL/ lymphoma and AML-type regimens were $67 \%$ and $27 \%$, respectively $(p=0.02)$. In our study, seven patients who received hyperCVAD or VPDL showed an objective response (five patients, CR). Responses were relatively long-lived, with a median length of 11.2 months (range, 6.2 to 19.4), compared with those previously reported for CHOP-like regimens [20]. Nevertheless, all patients showed relapse or disease progression (Table 3). Thus, regimens such as hyperCVAD and VPDL did not appear to be sufficient to ensure durable long-term remission. Allogenic SCT with myeloablative conditioning (MAC) has been shown to induce durable disease control leading to cure $[11,20]$. A study analyzing 34 patients who underwent allogenic SCT reported a 3-year disease-free survival and OS of $33 \%$ and $41 \%$, respectively. They also reported that long-term disease control was only observed after MAC, suggesting that MAC allogeneic SCT is an effective treatment. However, it is not clear to what extent intensive induction chemotherapy contributed to the success of the above study. Because BPDCN occurs in patients of advanced age and/or with comorbidities, MAC conditioning might not be suitable for elderly pa- 
tients. A few case series described the benefit of autologous SCT or reduced intensity conditioning allogeneic SCT $[21,22]$. A recent study comparing 11 patients who underwent autologous SCT and 14 patients who underwent allogeneic SCT in CR1 showed that the OS rates at 4 years for patients who underwent autologous SCT and allogeneic SCT were $82 \%$ and $53 \%(p=0.11)$ with a median follow-up of 53.5 months [15]. Thus, high-dose chemotherapy itself might provide a survival benefit for patients with BPDCN. In our study, one patient (\#2) with repeated relapses was able to maintain a CR after intensified chemotherapy, MEC (Table 3). However, the role of autologous SCT should be elucidated compared to allogeneic SCT.

The prognosis of patients with BPDCN is very poor with a median OS of about 12 to 14 months $[9,10,12,20]$. Several prognostic factors, including presentation with only localized skin lesions, Ki-67 proliferative index, TdT, and biallelic loss of locus 9p21, have been proposed in terms of survival $[10,20,23]$. In our study, two patients with only cutaneous involvement maintained longer remission compared to patients with involvement of multiple lymph nodes; however, patients presenting with only localized cutaneous lesions did not exhibit superior OS. Whether patients with only cutaneous lesions exhibit a better response to chemotherapy and prolonged survival remains unclear. In our cases, Ki- 67 and expression of TdT were not related to response to first-line chemotherapy or OS. Herein, we summarized the clinical features and outcomes of 10 patients with BPDCN. Isolated cutaneous lesions are frequently the first symptom, but they eventually disseminate. All patients treated with hyperCVAD or VPDL showed an objective response, but the above regimens do not appear sufficient for inducing long-term remission. Although the small number of patients in our study precludes drawing definitive conclusions, the development of more effective induction as well as consolidation treatment strategy should be warranted to improve this rare disease entity.

\section{KEY MESSAGE}

1. BPDCN (blastic plasmacytoid dendritic cell neoplasm) is rare and fetal disease, and there is no consensus on the optimal therapy.
2. Intensified chemotherapy with or without stem cell transplantation showed an objective response, but the duration of response was relatively short.

3. More effective induction as well as consolidation treatment strategy should be warranted.

\section{Conflict of interest}

No potential conflict of interest relevant to this article was reported.

\section{Acknowledgments}

This study was supported by grants from the Samsung Biomedical Research Institute (GL1B30311), and the National Research Foundation of Korea's (NRF) Basic Science Research Program (NRF-2014R1A2A1A11049853), which is funded by the Ministry of Education, Science and Technology.

\section{REFERENCES}

1. Chaperot L, Bendriss N, Manches O, et al. Identification of a leukemic counterpart of the plasmacytoid dendritic cells. Blood 2001;97:3210-3217.

2. Jegalian AG, Facchetti F, Jaffe ES. Plasmacytoid dendritic cells: physiologic roles and pathologic states. Adv Anat Pathol 2009;16:392-404.

3. Petrella T, Comeau MR, Maynadie M, et al. 'Agranular $\mathrm{CD}_{4}+\mathrm{CD}_{5} 6+$ hematodermic neoplasm' (blastic NK-cell lymphoma) originates from a population of $\mathrm{CD}_{5} 6+$ precursor cells related to plasmacytoid monocytes. Am J Surg Pathol 2002;26:852-862.

4. Willemze R, Jaffe ES, Burg G, et al. WHO-EORTC classification for cutaneous lymphomas. Blood 2005;105:3768-3785.

5. Facchetti F, Jones DM, Petrella T. Blastic plasmacytoid dendritic cell neoplasm. In: Swerdlow SH, Campo E, Harris NL, et al., eds. WHO Classification of Tumours of Haematopoietic and Lymphoid Tissues. 4th ed. Lyon: IARC Press, 2008;145-147.

6. Bueno C, Almeida J, Lucio P, et al. Incidence and characteristics of $\mathrm{CD}_{4}(+) / \mathrm{HLA}$ DRhi dendritic cell malignancies. Haematologica 2004;89:58-69.

7. Petrella T, Bagot M, Willemze R, et al. Blastic NK-cell lymphomas (agranular $\mathrm{CD}_{4}+\mathrm{CD}_{5} 6+$ hematodermic neo- 
plasms): a review. Am J Clin Pathol 2005;123:662-675.

8. Riaz W, Zhang L, Horna P, Sokol L. Blastic plasmacytoid dendritic cell neoplasm: update on molecular biology, diagnosis, and therapy. Cancer Control 2014;21:279-289.

9. Feuillard J, Jacob MC, Valensi F, et al. Clinical and biologic features of $\mathrm{CD}_{4}(+) \mathrm{CD}_{5} 6(+)$ malignancies. Blood 2002;99:1556-1563.

10. Julia F, Dalle S, Duru G, et al. Blastic plasmacytoid dendritic cell neoplasms: clinico-immunohistochemical correlations in a series of 91 patients. Am J Surg Pathol 2014;38:673-680.

11. Dalle S, Beylot-Barry M, Bagot M, et al. Blastic plasmacytoid dendritic cell neoplasm: is transplantation the treatment of choice? Br J Dermatol 2010;162:74-79.

12. Pagano L, Valentini CG, Pulsoni A, et al. Blastic plasmacytoid dendritic cell neoplasm with leukemic presentation: an Italian multicenter study. Haematologica 2013;98:239-246.

13. An HJ, Yoon DH, Kim S, et al. Blastic plasmacytoid dendritic cell neoplasm: a single-center experience. Ann Hematol 2013;92:351-356.

14. Suzuki R, Suzumiya J, Nakamura S, et al. Hematopoietic stem cell transplantation for natural killer-cell lineage neoplasms. Bone Marrow Transplant 2006;37:425-431.

15. Aoki T, Suzuki R, Kuwatsuka Y, et al. Long-term survival following autologous and allogeneic stem cell transplantation for blastic plasmacytoid dendritic cell neoplasm. Blood 2015;125:3559-3562.

16. Lucio P, Parreira A, Orfao A. CD12zhi dendritic cell lymphoma: an unusual case of non-Hodgkin lymphoma. Ann Intern Med 1999;131:549-550.
17. Vardiman JW, Thiele J, Arber DA, et al. The 2008 revision of the World Health Organization (WHO) classification of myeloid neoplasms and acute leukemia: rationale and important changes. Blood 2009;114:937-951.

18. Kharfan-Dabaja MA, Lazarus HM, Nishihori T, Mahfouz RA, Hamadani M. Diagnostic and therapeutic advances in blastic plasmacytoid dendritic cell neoplasm: a focus on hematopoietic cell transplantation. Biol Blood Marrow Transplant 2013;19:1006-1012.

19. Petrella T, Facchetti F. Tumoral aspects of plasmacytoid dendritic cells: what do we know in 2009? Autoimmunity 2010;43:210-214.

20. Reimer P, Rudiger T, Kraemer D, et al. What is $\mathrm{CD}_{4}+\mathrm{CD}_{5} 6+$ malignancy and how should it be treated? Bone Marrow Transplant 2003;32:637-646.

21. Dietrich S, Andrulis M, Hegenbart U, et al. Blastic plasmacytoid dendritic cell neoplasia (BPDC) in elderly patients: results of a treatment algorithm employing allogeneic stem cell transplantation with moderately reduced conditioning intensity. Biol Blood Marrow Transplant 2011;17:1250-1254.

22. Sanada Y, Nakazato T, Mihara A, et al. Sustained complete remission in an elderly patient with a blastic plasmacytoid dendritic cell neoplasm following autologous peripheral blood stem cell transplantation. Ann Hematol 2013;92:1285-1286.

23. Lucioni M, Novara F, Fiandrino G, et al. Twenty-one cases of blastic plasmacytoid dendritic cell neoplasm: focus on biallelic locus 9p21.3 deletion. Blood 2011;118:4591-4594. 